$$
\begin{aligned}
& \text { Submitel } 1 / 10 / 03 \\
& E C D-52-17-2003
\end{aligned}
$$

\title{
HEALTH MONITORING OF THERMAL BARRIER COATINGS BY MID-INFRARED REFLECTANCE
}

J.I. Eldridge, C.M. Spuckler, J.A. Nesbitt, and K.W. Street

NASA Glenn Research Center

Cleveland, $\mathrm{OH} 44135$

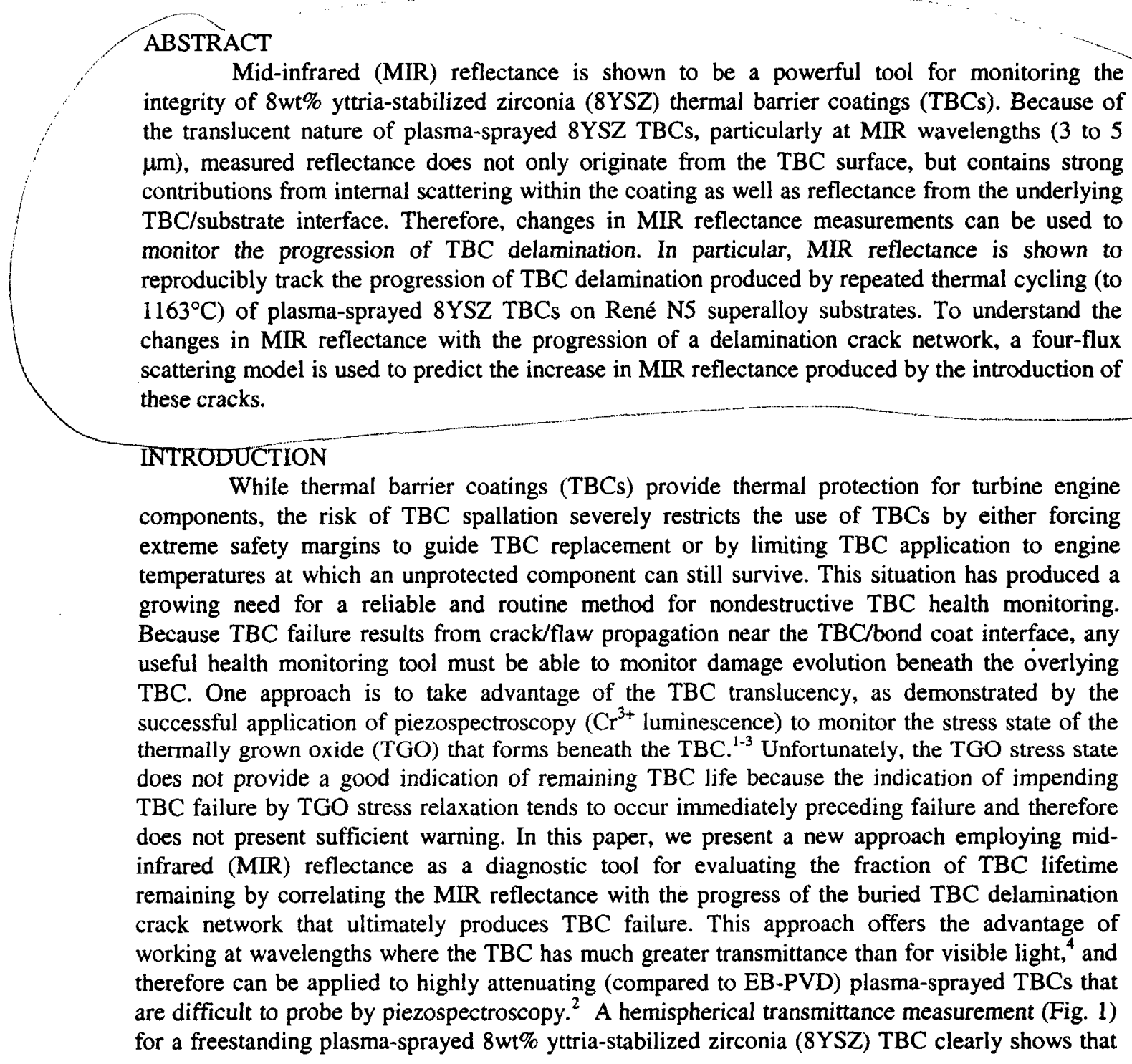




\section{Health Monitoring of \\ Thermal Barrier Coatings \\ by Mid-Infrared Reflectance}

J. I. Eldridge, C.M. Spuckler, J.A. Nesbitt, and K.W. Street

NASA Glenn Research Center

Cleveland, $\mathrm{OH}$

27th Annual Cocoa Beach Conference January 29, 2003 


\section{Motivation}

- Risk of TBC failure restricts application of TBCs

- Requires extreme safety margins for TBC replacement

or

- Limited to temperatures at which unprotected component can survive

- Reliable TBC health monitoring will overcome these restrictions. 


\section{Background}

- Any TBC health monitoring tool (optical, electrical, acoustic) must be able to monitor buried damage evolution.

- Optical techniques most amenable to non-contact monitoring.

- Piezospectroscopy ( $\mathrm{Cr}^{3+}$ luminescence) has been demonstrated to monitor stress state in thermally grown oxide (TGO) beneath TBC. (U. California-Santa Barbara, U. Connecticut)

- Very difficult to obtain luminescence signal through highly attchating klasma-cprayed TBCs.

- Measured TGO stress relaxation occurs immediately preceding or simultaneous with TBC; failure; therefore does not provide early warning.

- Mid-infrared (MIR) reflectance examind for advantages as TEC health monitoring tool.

- Uses wavelengths where TBC has maximum transmittance.

- Potentially more sensitive to earlier stages of TBC failure. 


\section{Maximum Transmittance at MIR Wavelengths}

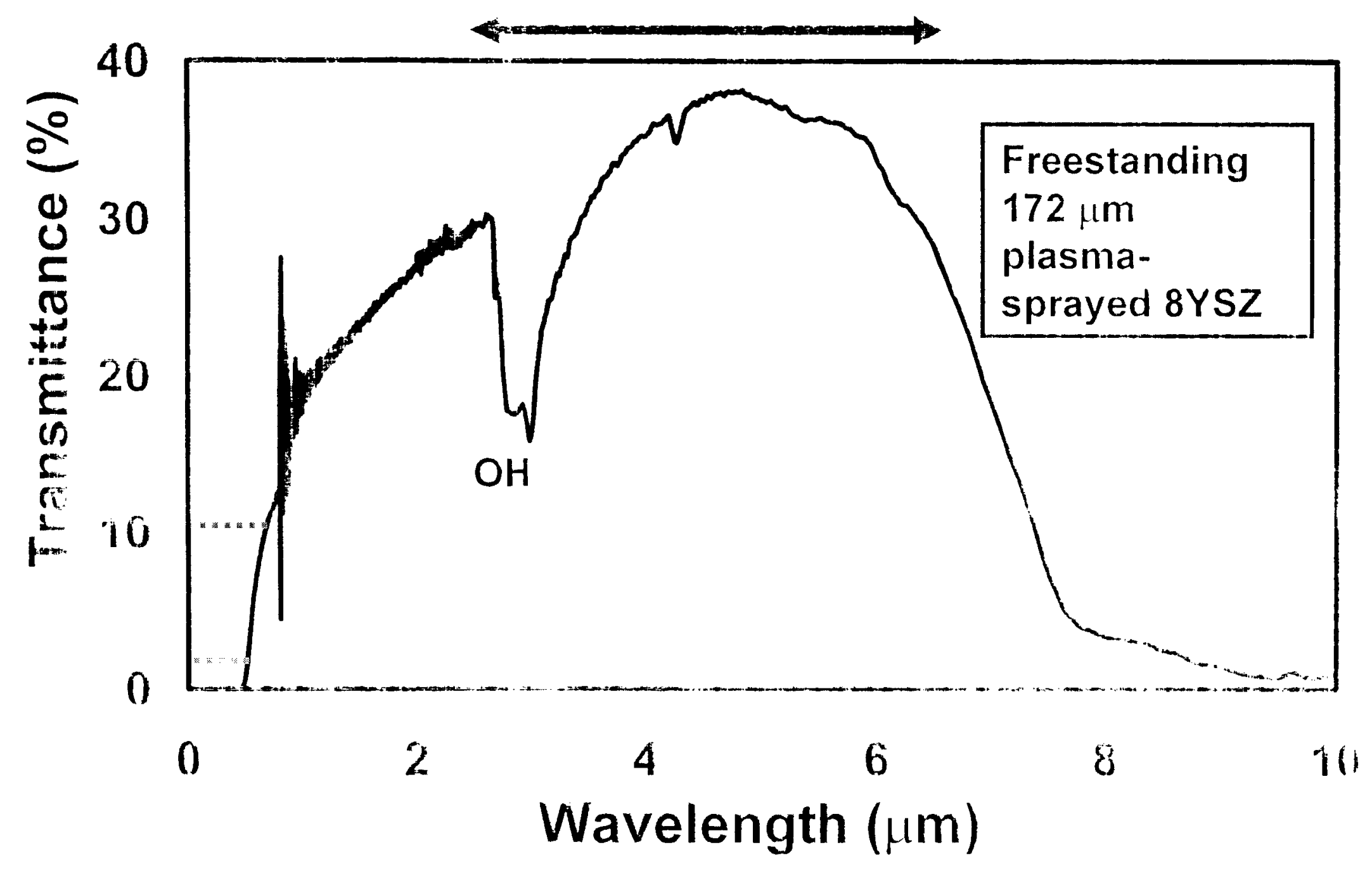




\section{Experimental Approach}

- Two types of TBC specimens:

- with bond coat (4)

- $200 \mu \mathrm{m}$ atmospheric plasma-sprayed 8YSZ

- $120 \mu \mathrm{m}$ low-pressure plasma-sprayed NiCrAIY bond coat

- René N5 superalloy substrate

-. no bond coat (3)

- $200 \mu \mathrm{m}$ atmospheric plasma-sprayed 8YSZ

- René N5 superalloy substrate:

- Monitor changes in MIR hemispherical reflectance with interrupted furnace cycling.

- 1 cycle $=45 \min \left(\mathrm{g} 1163^{\circ} \mathrm{C}+15 \mathrm{~min}\right.$ couling to $121^{\circ} \mathrm{C}$, in air

-.. Measue Mik reflectance atte $1^{\text {st }}$ cycle \& every $10^{\text {in }}$ cycle.

- Inspect for TBC failure (>20\% delamination) 


\section{Directional Hemispherical Reflectance Measurements}

integrating sphere accessory inside FTIR spectrometer

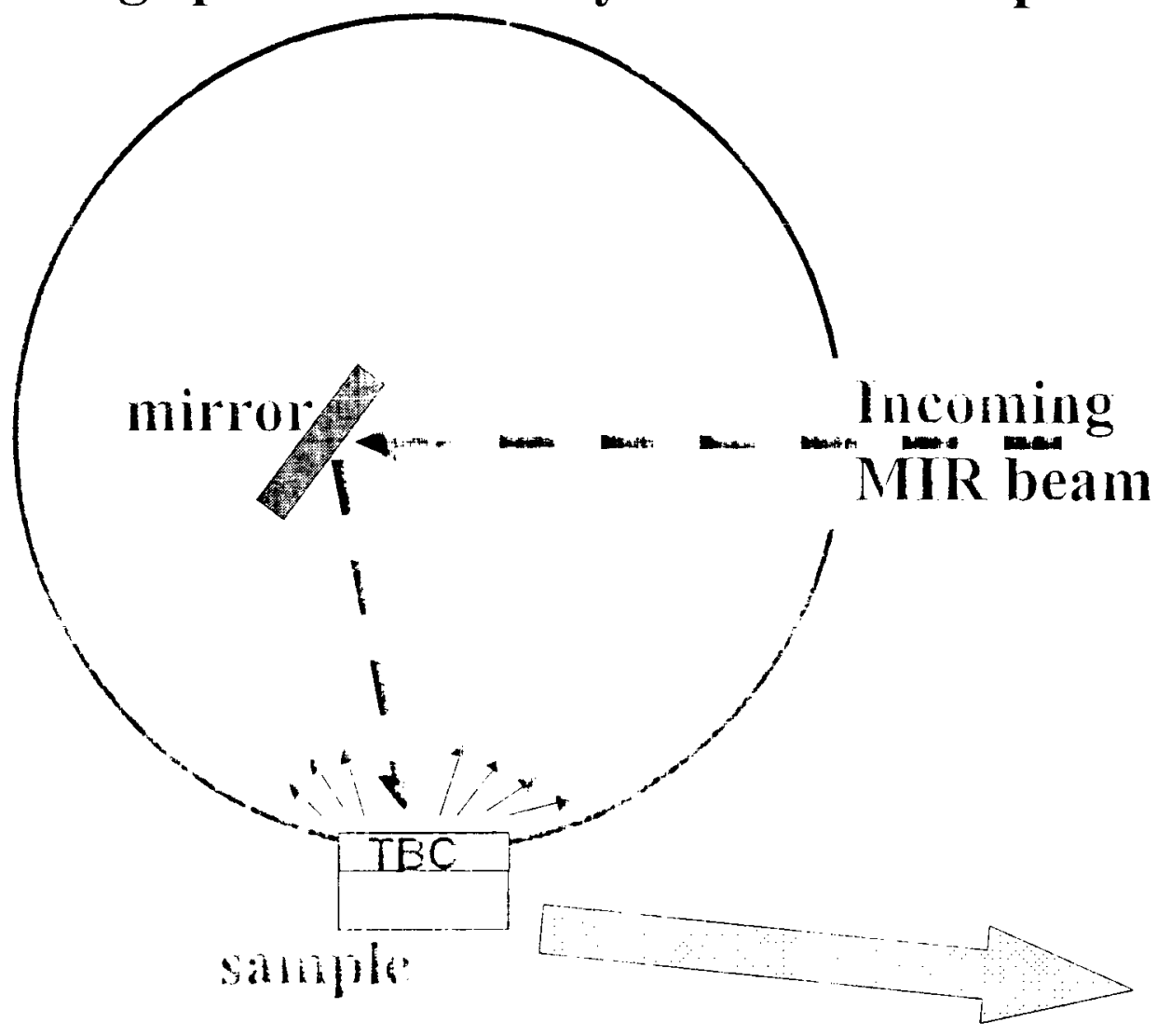

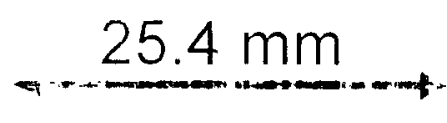

TEC 


\section{Effect of Furnace Cycling on Hemispherical Reflectance}

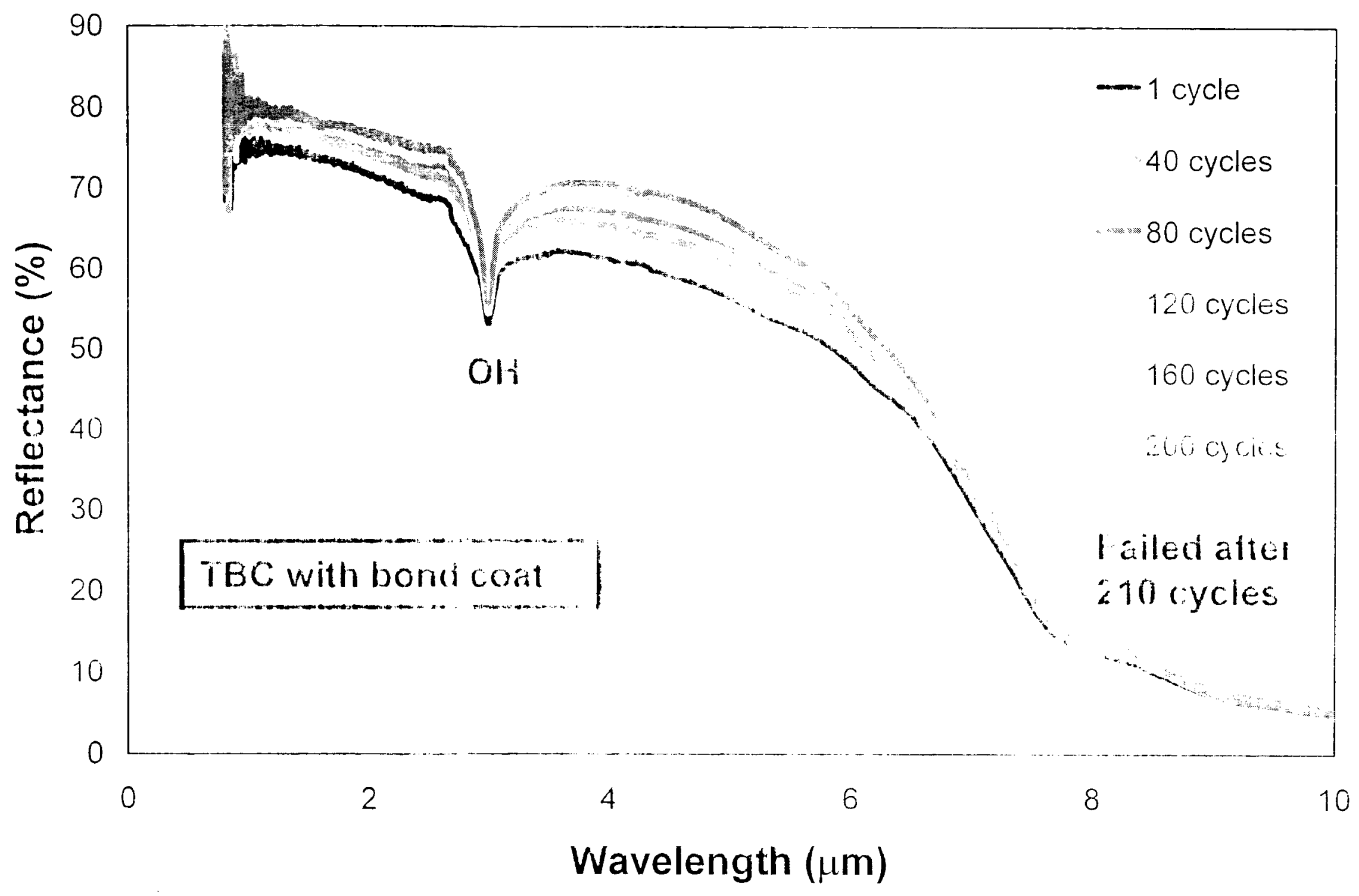




\section{Effect of Furnace Cycling on Hemispherical Reflectance \\ (expanded scale)}

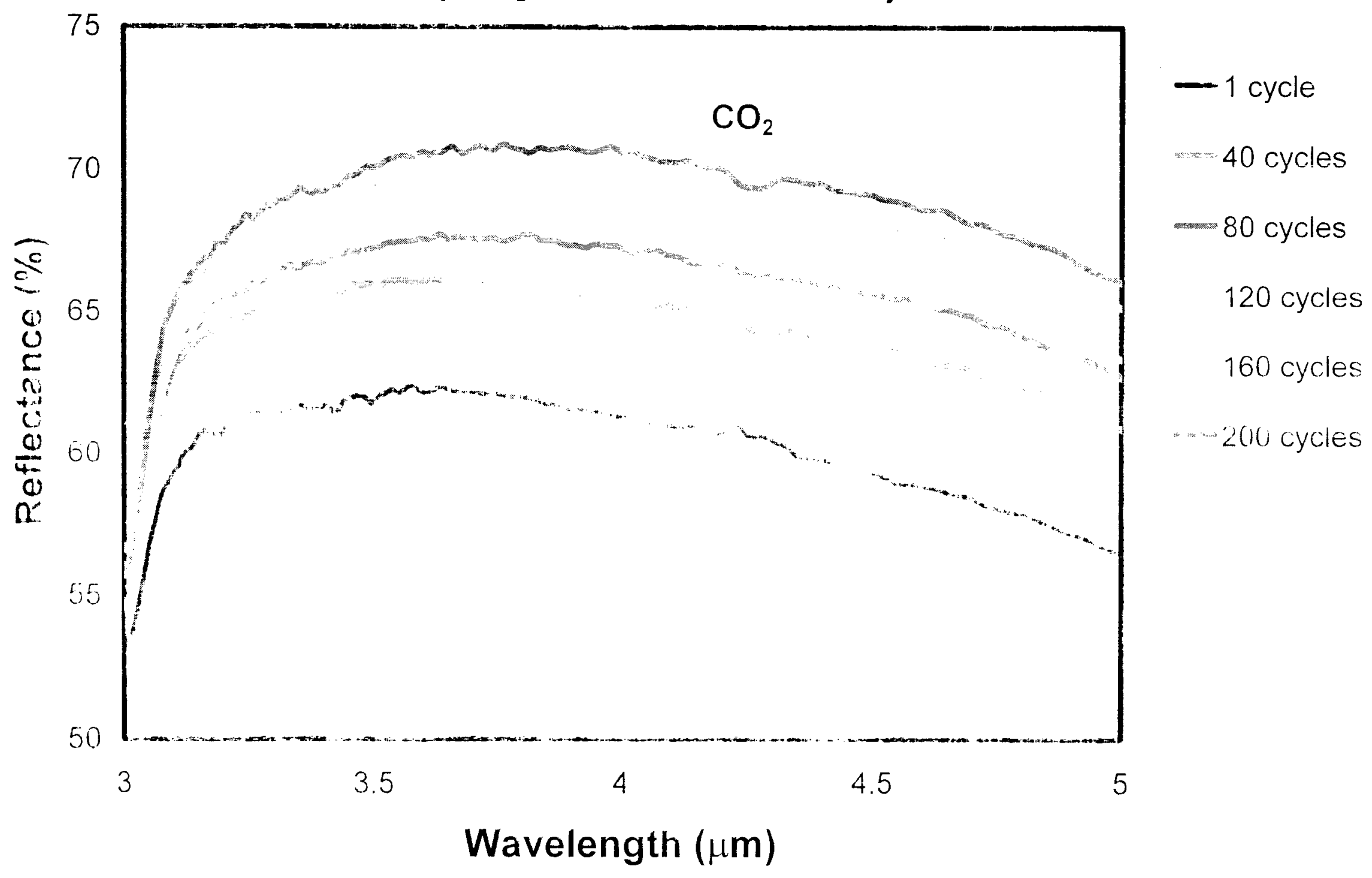




\section{OH Absorption Peak Interference}

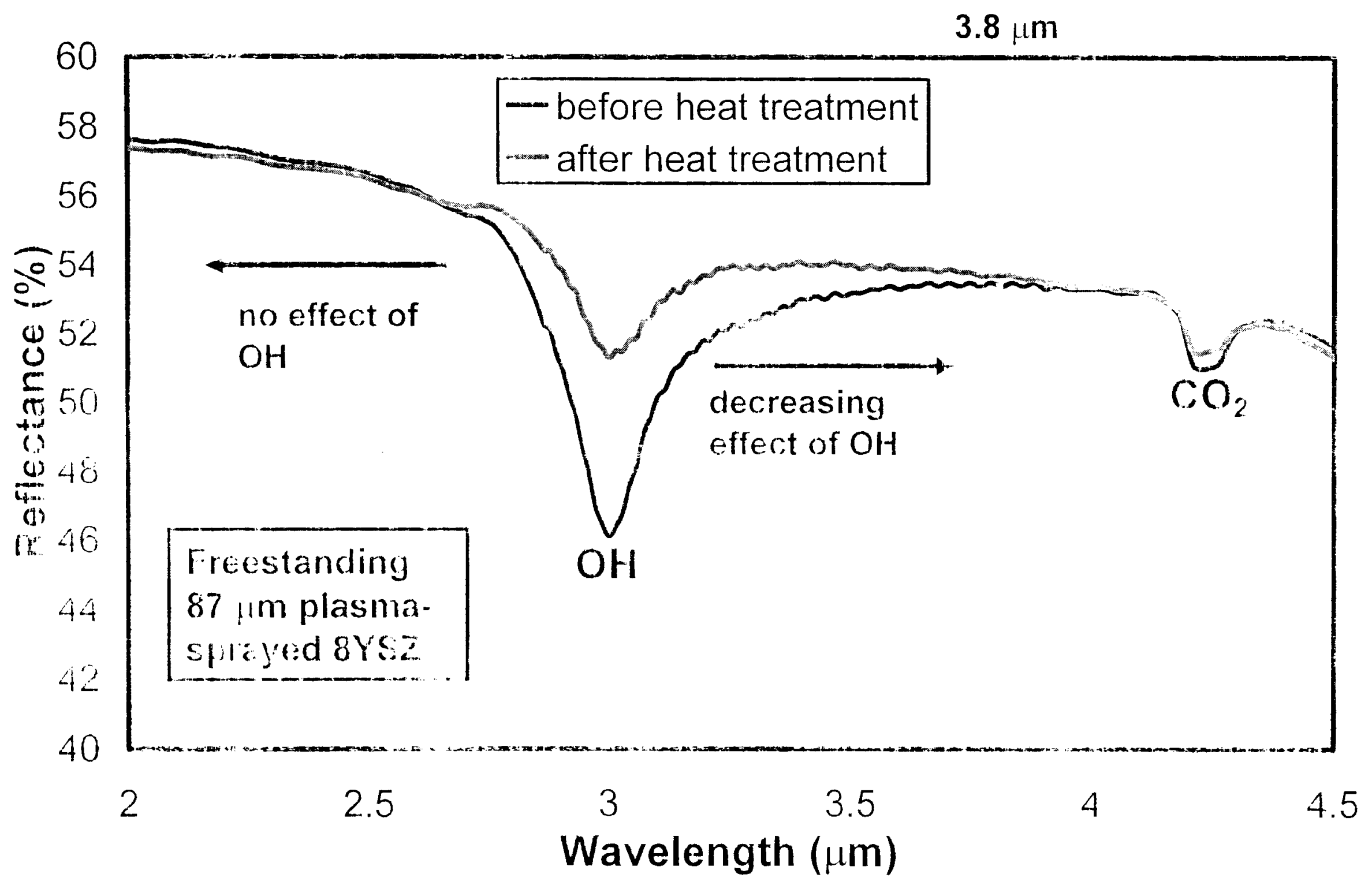


Hemispherical Reflectance $(\lambda=3.8 \mu \mathrm{m})$ Monitors Progress of TBC Delamination

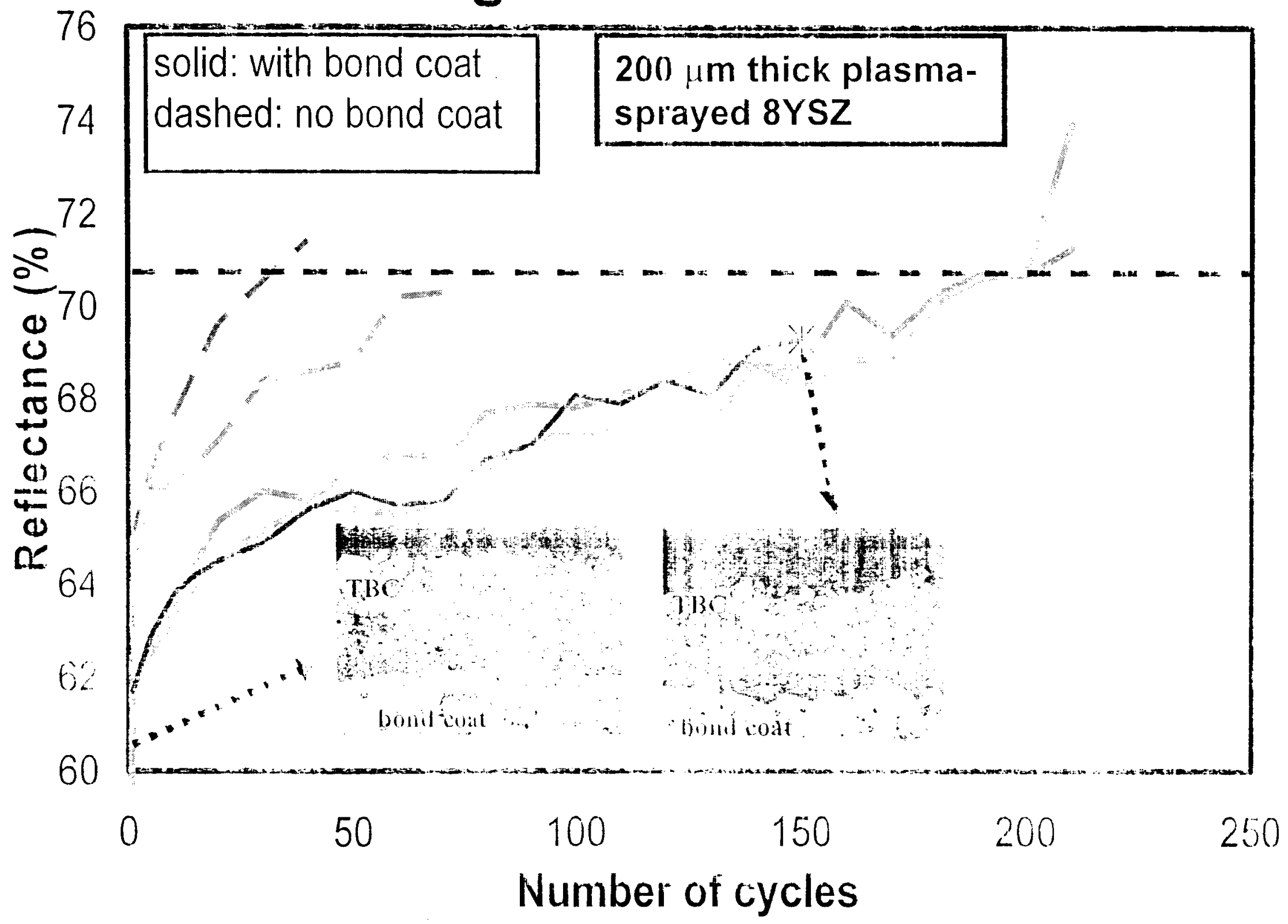




\section{Delamination crack network formed after 150 cycles}

Gradual progression allows effective health monitoring

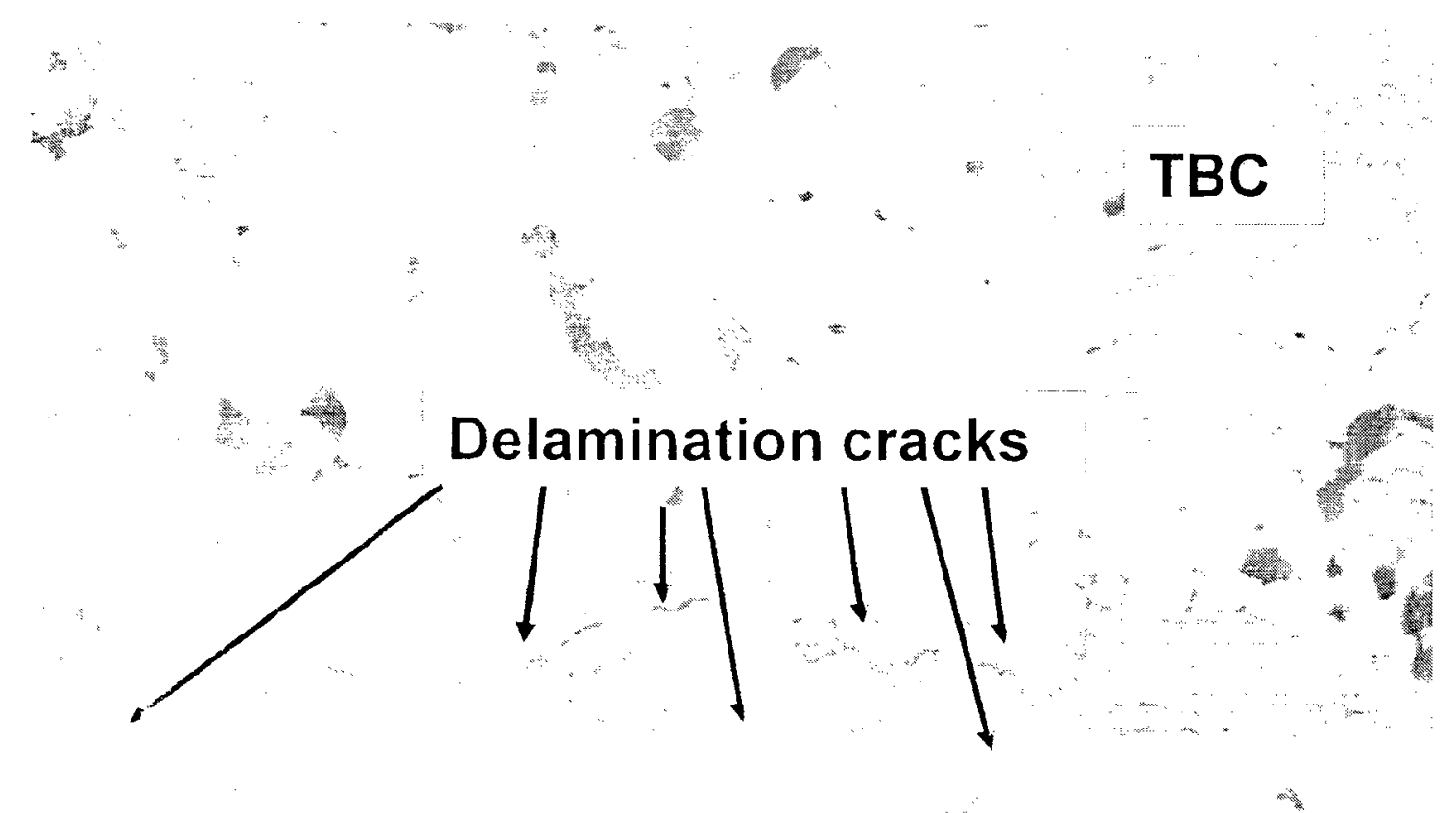

bond coat

$\$$

Rene N5 substrate

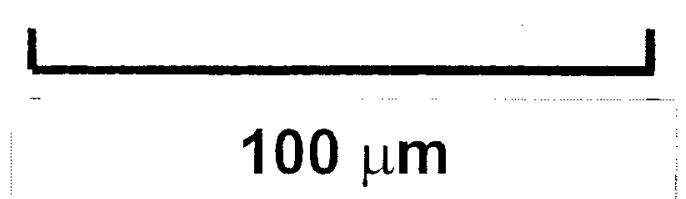




\section{4-Flux (Zero Absorption) Model for Normal Hemispherical Reflectance} TBC with underlying crack
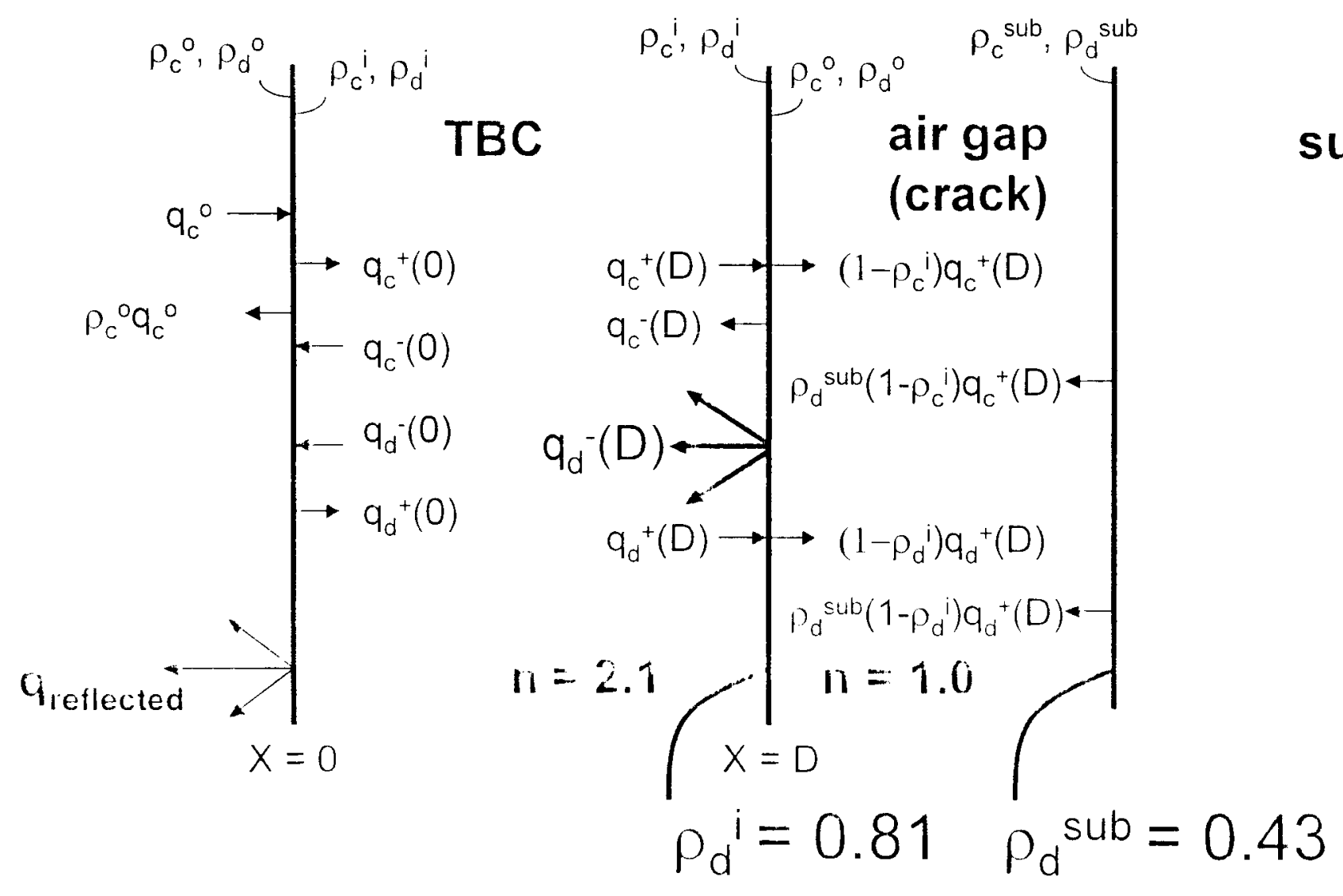

Crack introduces high internal reflection due to index of refraction change across TBC/crack interface.

$$
q_{\text {reflected }}=\rho_{c}^{0} q_{c}^{0}+\left(1-\rho_{c}^{i}\right) q_{c}^{-}(0)+\left(1-\rho_{d}^{i}\right) q_{d}^{-}(0)
$$


Predicted Effect of Crack on Hemispherical Reflectance (based on zero-absorption four-flux model)

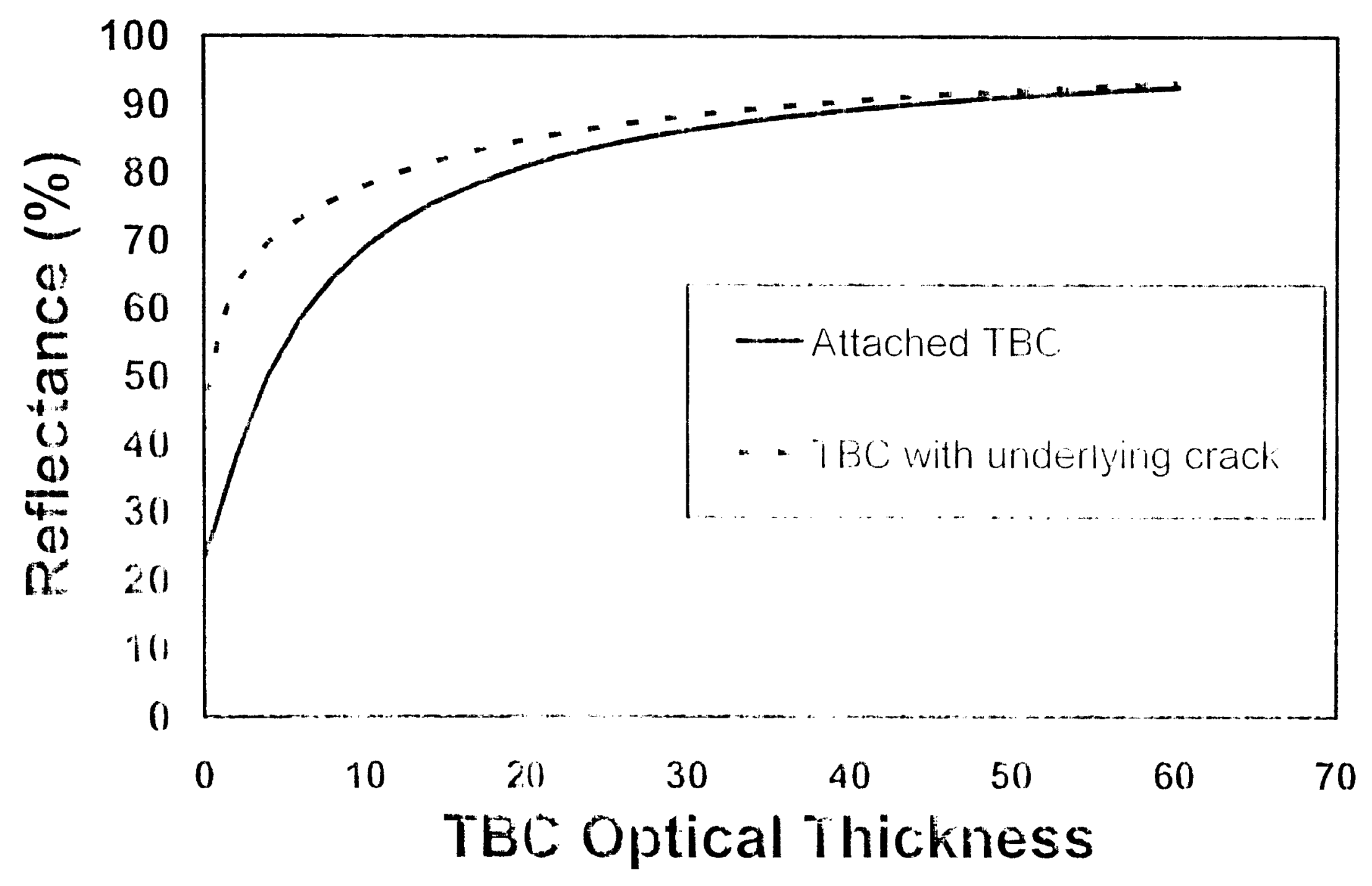




\section{Competing Influences on Hemispherical Reflectance}

- OH content in TBC

- TBC thickness variation

- $T B C$ siriering

- Thermally grown oxide (TGO) growth 


\section{Hemispherical Reflectance Increases with TBC Thickness}

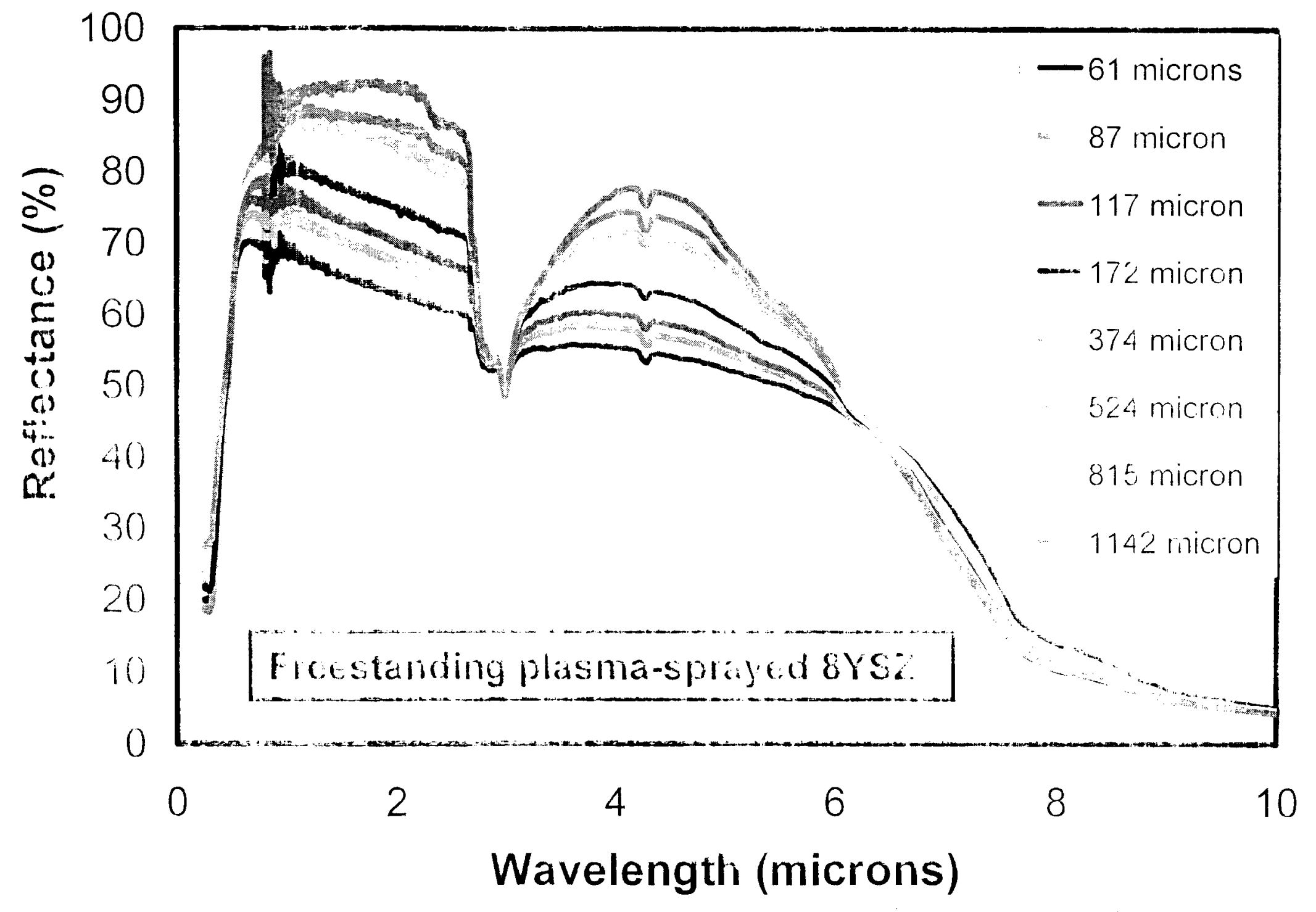




\section{Sintering Decreases Hemispherical Reflectance $160-\mu \mathrm{m}$-thick freestanding plasma-sprayed $8 \mathrm{YSZ}$}

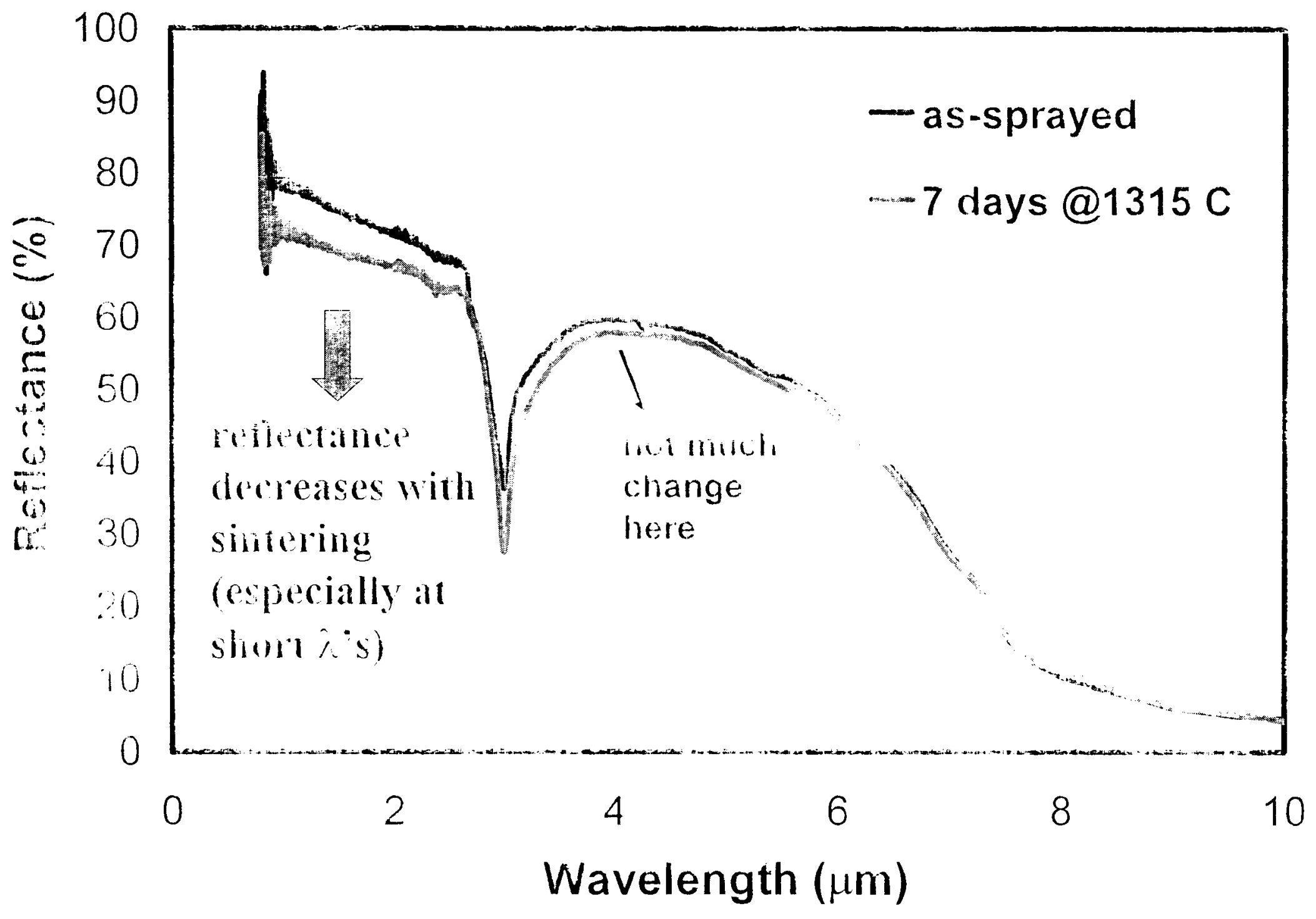




\section{Effect of Thermally Grown Oxide (TGO) on Hemispherical Reflectance}

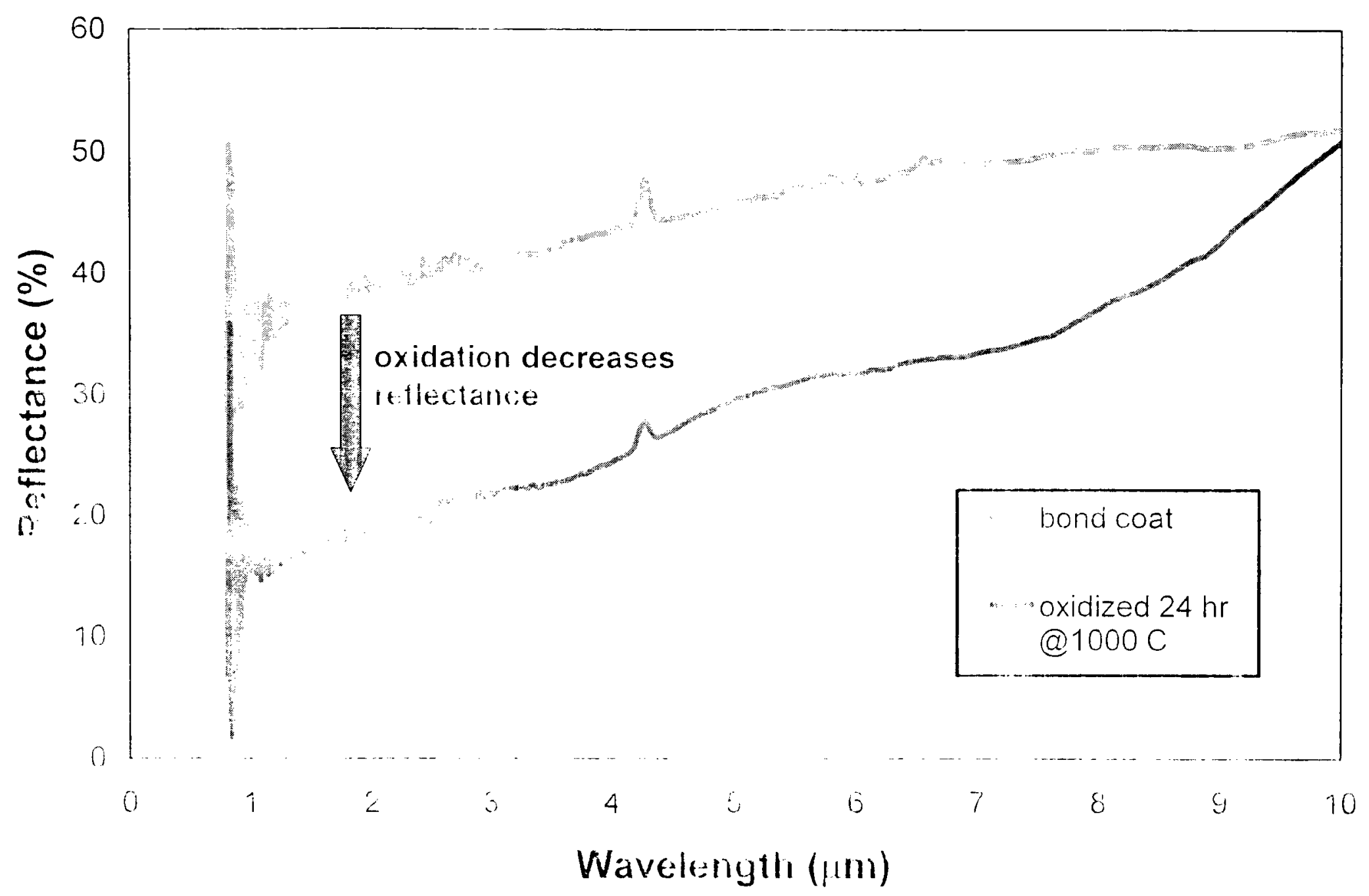

- TGO growth on bare substrate decreases reflectance.

- TGO growth beneath $\mathrm{TBC}$ requires further investigation. 


\section{Summary of Influences on MIR Hemispherical Reflectance}

- Increases Reflectance

- Progression of delamination crack network

- Decrease in $\mathrm{OH}$ content (can select wavelengths where effect is minimized)

- Decreases Reflectance

- Erosion (decrease in TE:C thickness)

- Sintering (preferentially affects shorter wavelengths)

- Requires Further inves igation

- Effect of buried $T(x)$ growth (cliange iri substrate ieflectance)

- Sintering \& erosion are noritactors 8 OH effect is

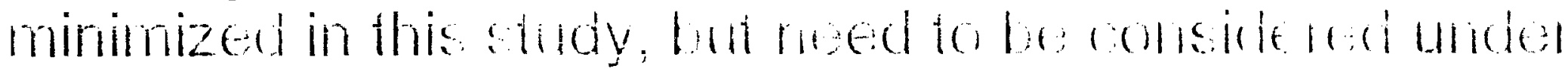
less controlled conditions.

- Competing influences have distinguishing spectral eftect "signatures" 


\section{MIR Reflectance Imaging Provides Practical Inspection Capability}

(provided by Rich Martin, Cleveland State University)

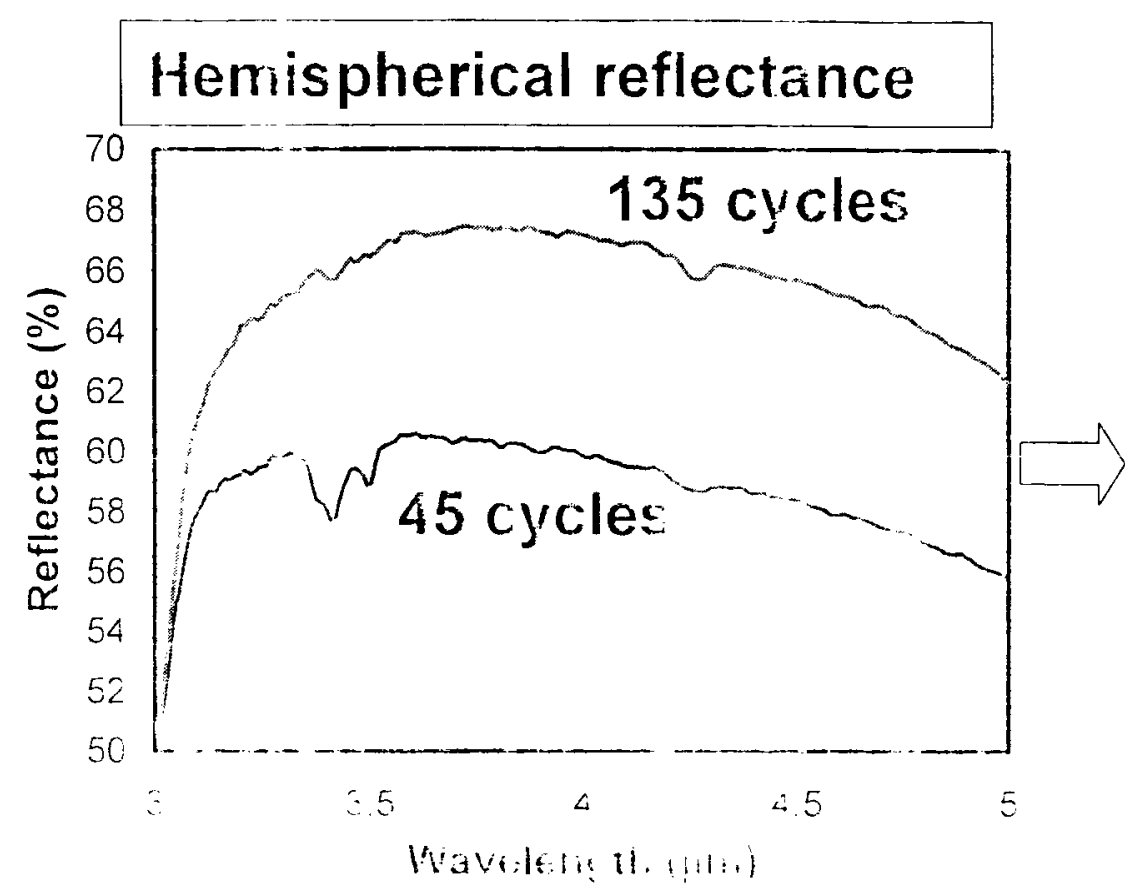

- Tliese are unoptinized ciuich \& diri) inages.

- In a giric provides localized fléw de it dibricipatiling.

- Visuál inspection by MIR reflectance imaging extends technique from lab to practical health-monitoring tool.

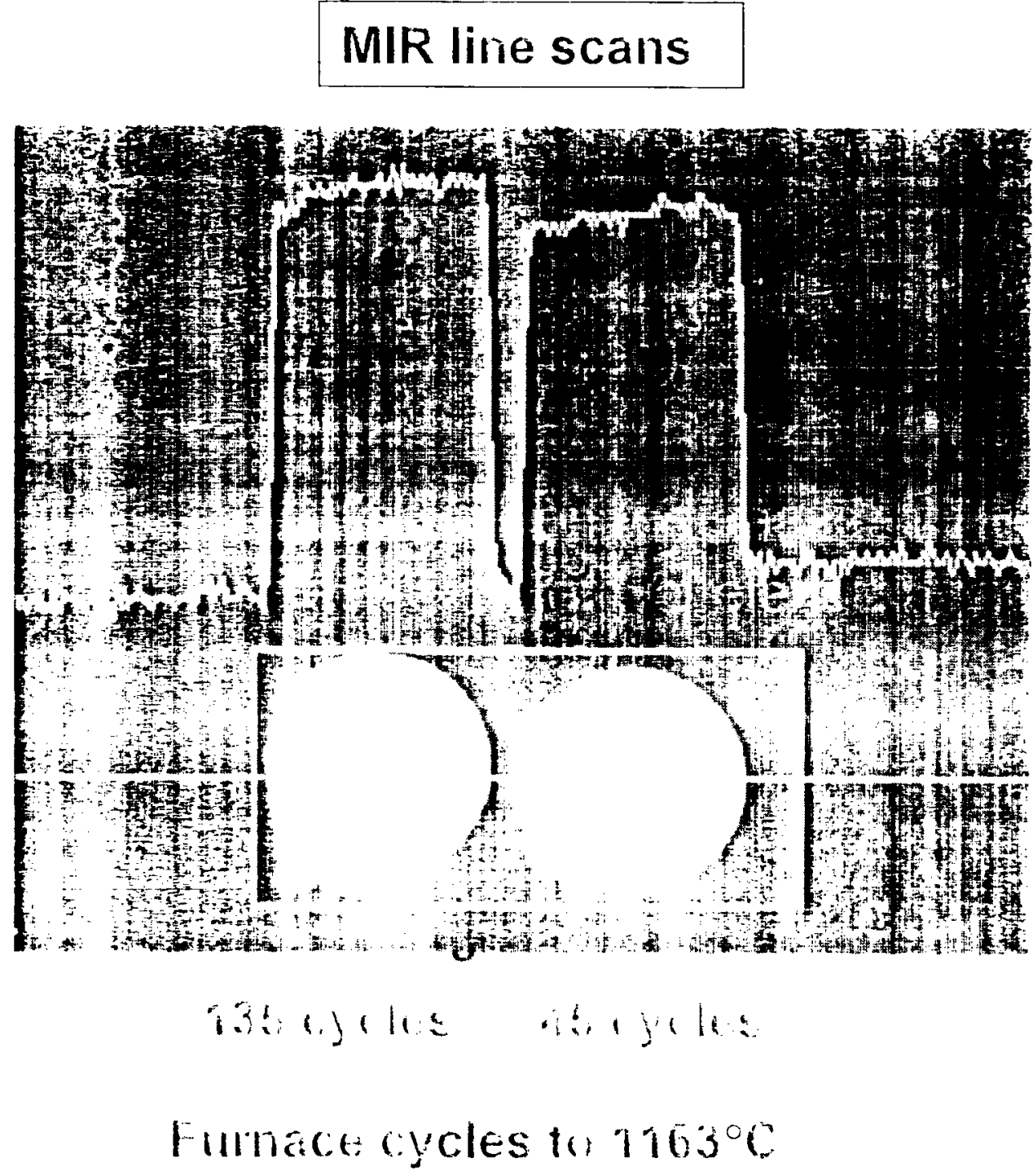




\section{Conclusions}

- MIR reflectance provides promising approach for TBC health monitoring.

- MIR wavelengths successfully probe through highly attenuating plasma-sprayed TBCs.

- MIR reflectance is sensitive to early stages of TBC failure.

- Gradual progression of delamination crack network allows early warning.

- Competing influences on MIR reflectance have distinguishing ipectral eftects.

- Visual inspection by MIR reflectance imaging will extend techrique from lat to practical health monitoring tool. 


\section{Acknowledgments}

- Chuck Barrett - furnace cycling

- George Leissler \& Sandy Leissler - spraying TBC specimens

- Rich Martin - MIR reflertance imager 